\title{
Bronchoscopic Intervention Obviates the Need for Continued Mechanical Ventilation in Patients with Airway Obstruction and Respiratory Failure from Inoperable Non-Small-Cell Lung Cancer
}

\author{
Septimiu Murgu Stephanie Langer Henri Colt \\ University of California, Irvine, Irvine, Calif., USA
}

\section{Key Words \\ Non-small-cell lung cancer - Central airway obstruction • Bronchoscopy $\cdot$ Respiratory failure $\cdot$ Airway stents}

\begin{abstract}
Background: Patients with advanced non-small-cell lung cancer (NSCLC) and acute respiratory failure (ARF) from central airway obstruction (CAO) may be offered end-of-life care rather than intensive care treatment and palliative bronchoscopic intervention. Objectives: To determine whether bronchoscopic intervention could be immediately successful in restoring airway patency and obviate the need for continued mechanical ventilation in a homogeneous group of inoperable mechanically ventilated patients with ARF and CAO from NSCLC. Methods: A retrospective study of 12 consecutive intubated and mechanically ventilated patients with inoperable or unresectable CAO from NSCLC referred for therapeutic bronchoscopic intervention between January 2003 and December 2008. Outcome measures included time-to-postintervention extubation and survival. Procedural success was defined as successful restoration of airway patency, extubation and removal from mechanical ventilation within $24 \mathrm{~h}$ after bronchoscopic intervention. Results: Twelve intubated and mechanically ventilated patients were admitted to the ICU during the 6-year study period. Airway patency was restored in 11/12 (91\%) patients. Bronchoscopic
\end{abstract}

intervention resulted in immediate extubation and discontinuation of mechanical ventilation in 9/12 (75\%) patients. Overall median survival was 228 days (range 6-927). For the 9 patients extubated within $24 \mathrm{~h}$ after intervention, however, median survival was 313 days (range 6-927). Conclusions: Intubated patients with respiratory failure caused by CAO from NSCLC can be successfully and rapidly removed from mechanical ventilation after bronchoscopic interventions aimed at restoring airway patency. Median survival greater than 10 months justifies ICU hospitalization and referral for bronchoscopic treatment.

Copyright $\odot 2012$ S. Karger AG, Basel

\section{Introduction}

The annual cost of therapy and extent of care for patients with cancer is projected to increase from USD 104 billion to USD 173 billion in the next 15 years [1]. This trend, which could bankrupt the health care system, is not sustainable [2]. While generally proposed cost containment strategies include a reevaluation of the types and choice of drugs used for treatment, greater incorporation of supportive care strategies into treatment plans and a reevaluation of the frequency with which imaging studies are performed, various medical and surgical subspecialties are also called upon to explore their use of 
technology and management algorithms in the care of specific cancer populations [1].

In the realm of critical care medicine, the appropriate allocation of critical care beds is an essential component of managing scarce resources and controlling health care costs [3]. Studies show that more than $28 \%$ of all health care expenditures occur in the last 6 months of life [4], and despite the increased use of hospice and home palliative care services, a terminal ICU admission may consume $80 \%$ of all terminal hospitalization costs [5]. ICU utilization among older adults dying with advanced lung cancer in the USA continues to rise; in-hospital and 6-month mortality rates are 54 and $73 \%$, respectively, for nonsurgical stage IIIB and stage IV lung cancer patients admitted to the ICU [6]. For patients with malignant central airway obstruction (CAO), median and 1-year survival rates are reportedly as low as 3.4 months and 15\%, respectively [7].

Most patients with lung cancer die from systemic metastatic disease, yet over a third of patients with lung cancer receiving ICU care have a potentially reversible condition. Survival beyond 90 days in nonsurgical patients with lung cancer requiring ICU admission is only $37 \%$, with survival decreasing to $12 \%$ at 1 year $[8,9]$. Many patients develop complications associated with CAO [10], and, when acute respiratory failure (ARF) ensues, the prognosis is particularly poor $[8,10]$. When respiratory failure is caused by airway obstruction, only $25 \%$ of patients are successfully weaned off mechanical ventilation [10], although some patients with malignant CAO from a variety of tumors benefit from interventional bronchoscopic procedures aimed at restoring airway patency [11], obviating the need for continued mechanical ventilation $[12,13]$, providing time to initiate additional therapies and prolonging survival [14].

There is a paucity of literature addressing the benefit, or lack thereof, of bronchoscopic interventions in specific homogenous patient populations with cancer, ARF and $\mathrm{CAO}$, however, because CAO is caused by many types of tumors with differing clinical courses and prognoses [9]. Outcome studies of critically ill patients with CAO are further confounded by the inclusion of a small number of patients with multiple histological types of malignancy, including patients with primary lung cancer and extrathoracic cancers requiring various types of ventilatory support that range from high-flow oxygen masks to noninvasive positive pressure ventilation or endotracheal intubation with mechanical ventilation [12-15].

The Society of Critical Care Medicine currently recommends ICU admission for all patients with advanced cancer who have a reversible condition such as pulmonary embolism, cardiac tamponade or airway obstruction [3]. Accompanying this recommendation is a prioritization scale identifying those patients who will benefit most from ICU admission (Priority 1) from those who will not benefit at all (Priority 4). Patients with cancer complicated by airway obstruction are assigned a priority 3 , defined as 'unstable patients who are critically ill but have a reduced likelihood of recovery because of underlying disease or nature of their acute illness' [3]. While some of these patients may be intubated, placed on mechanical ventilation and considered for treatment of their $\mathrm{CAO}$, others may receive intensive care treatment only to relieve the effects of acute illness, pain or suffering. In these instances, it is likely that limits on therapeutic efforts are set, such as no intubation and no cardiopulmonary resuscitation [3].

To our knowledge, there are no studies of bronchoscopic interventions in a homogenous group of inoperable patients with primary lung cancer in whom results would either justify or refute a strategy of intubation and mechanical ventilation in the setting of CAO-induced respiratory failure. A case-control study would require a control group of non-small-cell lung cancer (NSCLC) patients with ARF and CAO who do not undergo therapeutic intervention. Such a study does not seem feasible in today's medical environment. The purpose of our descriptive case series analysis, therefore, was to simply determine whether therapeutic bronchoscopic intervention could be immediately successful in restoring airway patency and obviate the need for continued mechanical ventilation in a homogeneous group of inoperable intubated and mechanically ventilated patients with ARF and CAO from advanced NSCLC.

\section{Materials and Methods}

\section{Study Design}

The medical records and digital media (bronchoscopy videos and photos) of 12 consecutive patients with inoperable NSCLC, CAO and ARF admitted to the University of California Irvine Medical Center ICU between January 2003 and December 2008 were reviewed. The study was approved by the committee of the Center's institutional review board (protocol Nos. 2005-4236). All patients underwent rigid bronchoscopy in an attempt to restore airway patency according to our policy of offering bronchoscopic intervention to all patients admitted or transferred to our ICU for ARF due to CAO. Our protocol includes laser resection and debulking for endobronchial exophytic disease and/or stent insertion in case of extrinsic compression or residual obstruction greater than $50 \%$ after laser-assisted rigid bronchoscopic debulking. 


\section{Methods}

Hospital records and procedure notes were reviewed in order to extract patient age, gender, comorbidities and duration of respiratory failure before intubation. Also noted were the location and extent of airway obstruction, the performance of rigid bronchoscopy with tumor resection, laser or stent insertion, the number, type, size and location of stents placed, the occurrence of procedure-related complications such as new respiratory insufficiency requiring intubation, bleeding, perforation, pneumothorax, cardiac arrhythmias requiring medical intervention and intraoperative or immediate $(<48 \mathrm{~h})$ postoperative death. Rigid bronchoscopy was performed under general anesthesia using spontaneous assisted ventilation with an open system. Nd-YAG resection (Nd:YAG 1064; Laserscope, San Jose, Calif., USA) was performed using 20-40-W pulses and pulse durations of $0.5-$ $1.0 \mathrm{~s}$. Tumor debulking was performed using the beveled tip of the rigid bronchoscope and forceps. Flexible bronchoscopy was performed as necessary to help clear the airways of secretions and blood, and to assess the patency of distal bronchial segments. When indicated, silicone or self-expanding covered Ultraflex stents were inserted through the rigid bronchoscope (fig. 1). This was done using a specially designed stent introducer system (EFER rigid bronchoscope, Bryan Corp, Woburn, Mass., USA) or using a specially designed forceps (Rusch, Karl-Storz, Germany). Silicone airway stents were either studded (Bryan Corp), ringed (Hood, Woburn, Mass., USA) or Y silicone stents (Bryan Corp) (fig. 1). Ultraflex stents (Boston Scientific Inc., Natick, Mass., USA) were inserted by positioning the rigid bronchoscope proximal to the stenosis. A stent delivery catheter was advanced alongside the rigid telescope in order to deploy the stent under bronchoscopic visualization. Repositioning was done using grasping forceps. Patients were monitored for not more than $2 \mathrm{~h}$ in the postanesthesia care unit adjacent to the operating theater, after which they were transferred to either the ICU or the medical ward, based on their ventilatory status and need for observation or further therapy.

\section{Analysis}

A successful intervention was defined as one that technically restored airway patency and was accompanied by extubation and removal from mechanical ventilation within $24 \mathrm{~h}$ after intervention. Postintervention survival was also recorded. Patients were referred to our service strictly for bronchoscopic intervention, following which, they returned to their referring facility. It is our practice to follow up with patients and referring physicians by telephone, and to record the date and cause of death in our medical records when possible. Descriptive statistics are presented using mean and range for age and duration of respiratory failure prior to bronchoscopic intervention, and median and range for survival postintervention.

\section{Results}

Twelve intubated and mechanically ventilated patients with NSCLC and ARF caused by CAO were referred and admitted to the ICU for possible bronchoscopic intervention during the 6-year study period. Because of underly-
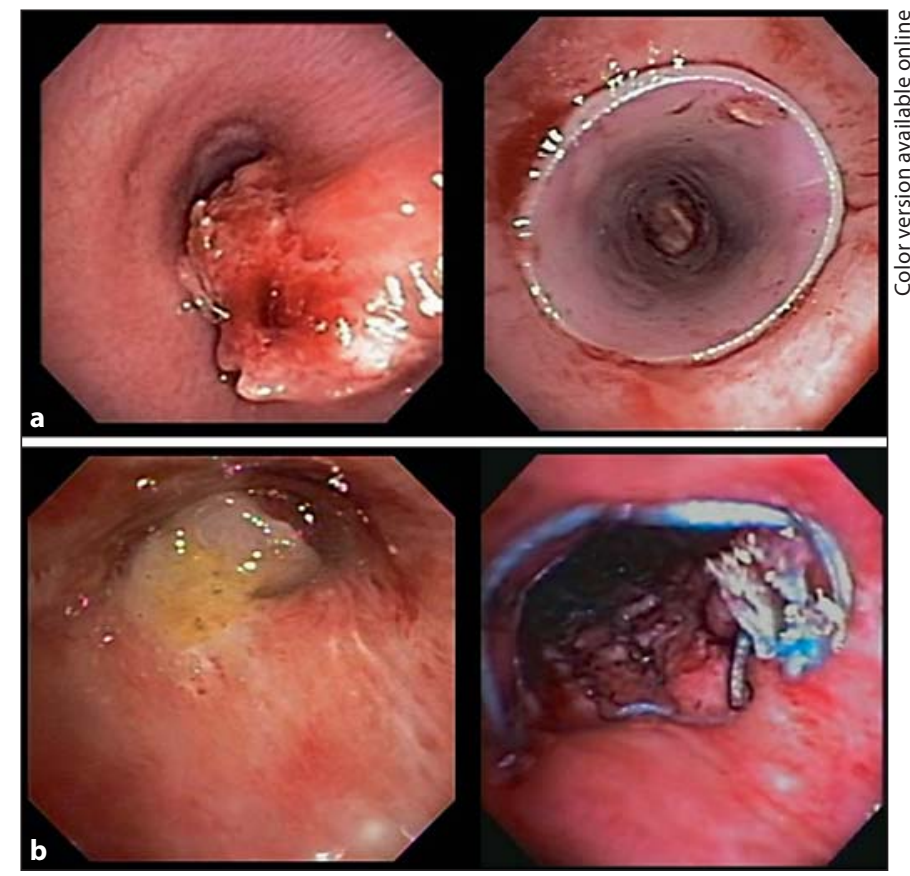

Fig. 1. a Severe tracheal obstruction from a poorly differentiated NSCLC before and after insertion of a $16 \times 50 \mathrm{~mm}$ straight studded silicone stent in the mid-lower trachea. $\mathbf{b}$ Complete distal left main bronchial obstruction from adenocarcinoma before and after insertion of a $12 \times 40 \mathrm{~mm}$ partially covered self-expendable metallic stent.

ing comorbidities, high surgical risk or the extent of airway abnormalities, none was considered a candidate for open surgical resection. According to our patient care policy, all patients were scheduled for rigid bronchoscopy under general anesthesia to attempt restoration of airway patency. Patient characteristics, bronchoscopic treatment modalities, extubation rate and survival are summarized in table 1 . Nine patients were males (75\%) and 3 were females (25\%). The mean patient age was 64.16 years (range 46-82). The mean duration of respiratory failure before intervention was 6 days (range 1-17). Karnofsky performance status was 20 or less for all patients. All had advanced NSCLC ( 2 patients were stage IV and 10 patients were stage IIIB) (table 1). Four had new diagnoses of primary lung cancer (patients 2, 4, 6 and 12), and 8 had recurrent and/or progressive disease. Prior to the onset of respiratory failure, 2 of the 12 had received chemotherapy (patients 1 and 3) and 2 combined chemoradiation (patients 5 and 7). Follow-up regarding treatment after bronchoscopic intervention showed that 3 patients had no additional therapy (patients 5, 7 and 9), 5 had combined 


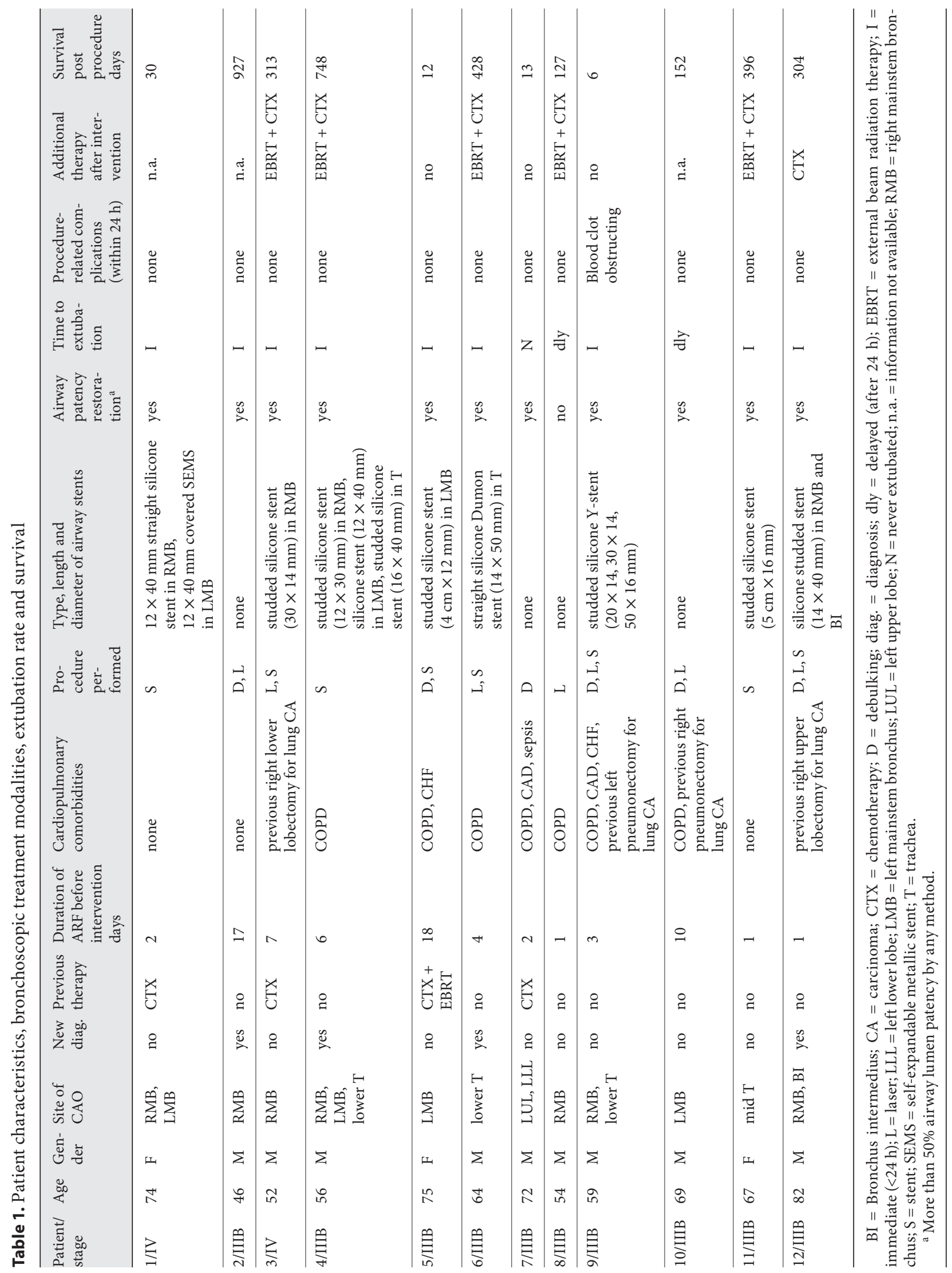


chemoradiation (patients $3,4,6,8$ and 11), 1 had chemotherapy alone (patient 12) and data were unavailable in 3 (patients 1, 2 and 10) (table 1). Airway patency was successfully restored in 11/12 (91\%) patients after 1 or more bronchoscopic interventions in a single setting. Airway stents were inserted in $8(66.6 \%)$ patients. Bronchoscopic interventions resulted in immediate extubation and discontinuation of mechanical ventilation in 9/12 (75\%) patients. Among the 3 patients in whom bronchoscopic intervention was not identified as successful, 1 was extubated after 8 days and 2 returned to their referring facilities on mechanical ventilation (1 was successfully extubated after completion of external beam radiation therapy). There were no intraoperative complications. One patient (case 9) required emergent flexible bronchoscopy $24 \mathrm{~h}$ after intervention in order to remove a blood clot partially occluding the airway stent. Patients 1, 5, 7 and 9 died within 30 days of the intervention: 1 from sepsis (patient 7) and another from massive hemoptysis (patient 9). The cause of death for patients 1 and 5 is unknown.

Median survival for the 12 patients overall was 228 days (range 6-927), and 313 days (range 6-927) for the 9 patients identified as having undergone a successful bronchoscopic procedure (i.e. resulting in extubation and removal from mechanical ventilation within $24 \mathrm{~h}$ after intervention).

\section{Discussion}

Grim outcomes for critically ill cancer patients requiring life support prompt many clinicians to limit life-prolonging treatment or to suggest refusing ICU admission [16-18]. In fact, while advances in systemic therapy and supportive management of organ dysfunction have improved survival, $40-70 \%$ of critically ill patients with advanced lung cancer and poor functional status admitted to the ICU go on to die in hospital, and for those who are discharged from hospital alive, median survival may be only 33 days [6]. Some might suggest that the economic costs of intensive care for patients with advanced cancer are prohibitive, and that by extending their lives, costs are greater than if they die at the time of their index admission.

ICU admission for respiratory failure necessitating invasive mechanical ventilation is a reality, however, and may be considered in as many as $40-70 \%$ of patients suffering from lung cancer $[19,20]$. Respiratory failure is usually caused by pneumonia, acute lung injury/acute respiratory distress syndrome, diffuse alveolar hemorrhage, airway bleeding, venous thromboembolism or
CAO. Mortality in this patient population increases with the number of failed organs, the severity of comorbidities and when airway obstruction is present [19]. In 1 study, hospital mortality was $83 \%$ in mechanically ventilated lung cancer patients with CAO compared to $62 \%$ in patients without an obstructed airway [19].

Over the last 20 years, studies have shown that bronchoscopic interventions aimed at restoring airway patency allow extubation and improve functional status in many critically ill patients with CAO. These studies included patients with various types of cancer and benign disease, requiring various levels of ventilatory support [12-15, 21, 22]. Colt and Harrell [12] described successful removal from ventilatory support in 10 of 19 patients (52.6\%) with both benign and malignant disease, concluding that if bronchoscopic interventions were unsuccessful, particularly in patients with cancer, supportive measures should be rapidly instituted. Shaffer and Allen [13] reported successful extubation 2-11 days after metal stent insertion in 6 of 8 patients with CAO, but $25 \%$ of the patients had a benign cause of airway obstruction. Lo et al. [22] successfully extubated all 7 patients in their study, although here too, 2 had respiratory failure from a benign cause of CAO. In a larger cohort, Jeon et al. [14] reported 36 patients with respiratory failure and malignant $\mathrm{CAO}$ from various tumors, noting that patients who underwent systemic or radiation therapy in addition to bronchoscopic intervention survived longer than those who underwent bronchoscopic palliation of CAO alone (median survival was 38.2 and 6.2 months, respectively). In a retrospective study from Holland, 14 patients with advanced disease from esophageal cancer $(n=5)$ or NSCLC $(n=9)$ had immediate symptomatic relief after interventional bronchoscopic intervention, prompting general practitioners responsible for terminal care at home to view airway stent insertion as a worthwhile procedure, and to recommend that bronchoscopic intervention be part of a treatment plan [23].

This reproducible evidence suggests that successful bronchoscopic restoration of airway patency in critically ill patients with malignant $\mathrm{CAO}$ from a variety of tumors improves symptoms, allows extubation, avoids suffocation, and in some patients, allows time for additional systemic therapies to take effect in order to prolong survival [21]. Results from our study support these conclusions and demonstrate their applicability specifically to patients with advanced NSCLC and CAO severe enough to prompt endotracheal intubation and mechanical ventilation. Considering that the majority of our patients were successfully extubated within $24 \mathrm{~h}$ after intervention, and 
survived a median of 313 days (range 6-927), a corollary of our results could be that NSCLC patients with ARF from $\mathrm{CAO}$ requiring endotracheal intubation and mechanical ventilation be admitted to the ICU for evaluation and bronchoscopic intervention to palliate airway obstruction. Without knowing how many patients with $\mathrm{ARF}$ and $\mathrm{CAO}$ were not referred, however, such a statement cannot be substantiated. Controlled studies to specifically address the benefits of intervention may not be ethically justifiable, but observational studies could address this issue in addition to evaluating quality of life after bronchoscopic intervention.

The limitations of our descriptive case series are mostly due to its retrospective nature and small sample size. Still, this is the largest study of its kind in this patient population. These limitations preclude a study of the potential effects of age, tumor stage and history of chemotherapy or radiation on outcomes and quality of life. Furthermore, conclusions must be tempered knowing that one's failure to restore airway patency or to immediately extubate a patient does not always signal imminent death. This was illustrated by our patient (case No. 8) in whom airway patency could not be restored bronchoscopically, but who responded favorably to external beam radiation therapy, resulting in extubation and 4 months of additional life. On the other hand, successful restoration of airway patency alone is not necessarily a guarantee that patients can be extubated. This was illustrated in another of our patients (case No. 7) who died as a consequence of pneumonia and sepsis while still on the ventilator 13 days after bronchoscopy. Engaging in end-of-life discussions, therefore, is obviously necessary in this patient population as a prerequisite to good planning [1]. Investigators have shown that $50 \%$ of patients with metastatic lung cancer had not had a discussion with their doctors about hospice 2 months before their death [24], and that the average length of stay in hospice for patients with lung can- cer is only 4 days [25]. ICU admission provides an opportunity, albeit expensive, to have these conversations, to reset expectations and to integrate palliative care services in an effort to reduce anxiety, modify the extent of care and improve family satisfaction [1].

These findings are relevant to oncologists, pulmonologists and intensivists caring for critically ill patients with advanced NSCLC. Determining prognosis in critically ill individuals has proven to be difficult and fraught with error [26]. Severity-of-illness scores, such as the Acute Physiology and Chronic Health Evaluation (APACHE), Simplified Acute Physiology Score (SAPS) and Mortality Probability Models (MPM) have fared poorly in critically ill cancer patients because of inadequate calibration and underestimation of mortality [26]. One proposed strategy consists of ICU admission with unrestricted care for a limited period of time (48-72 h) followed by reassessment. This study, however, included patients requiring ICU admission for shock, neurological complications and respiratory failure from a variety of causes: in only $16 \%$ (9 patients) was ARF prompted by airway obstruction [8]. Another strategy includes following SCCM prioritization guidelines for ICU admission and treatment alternatives. In this case, ARF may be considered reversible, but intubation and mechanical ventilation may not always be offered because of a 'reduced likelihood of recovery'.

While our conclusions pertain solely to patients with advanced NSCLC, until a prospective multi-institutional study replicates or refutes our results and the findings of other studies [21], we suggest that patients with NSCLC and ARF from CAO be considered to have a potentially reversible cause for respiratory failure. In these cases, intubation, mechanical ventilation and ICU admission are warranted while awaiting evaluation for palliative bronchoscopic interventions.

\section{References}

1 Smith TJ, Hillner BE: Bending the cost curve in cancer care. N Engl J Med 2011;364:2060 2065.

2 Bach PB: Limits on Medicare's ability to control rising spending on cancer drugs. N Engl J Med 2009;360:626-633.

3 Guidelines for intensive care unit admission, discharge, and triage. Task Force of the American College of Critical Care Medicine, Society of Critical Care Medicine. Crit Care Med 1999;27:633-638.
4 Seshamani M, Gray A: Time to death and health expenditure: an improved model for the impact of demographic change on health care costs. Age Ageing 2004;33:556-561.

5 Angus DC, Barnato AE, Linde-Zwirble WT, et al: Use of intensive care at the end of life in the United States: an epidemiologic study. Crit Care Med 2004;32:638-643.

6 Sharma G, Freeman J, Zhang D, et al: Trends in end-of-life ICU use among older adults with advanced lung cancer. Chest 2008; 133 : 72-78.
7 Lemaire A, Burfeind WR, Toloza E, et al: Outcomes of tracheobronchial stents in patients with malignant airway disease. Ann Thorac Surg 2005;80:434-438.

8 Toffart AC, Minet C, Raynard B, et al: Use of intensive care in patients with nonresectable lung cancer. Chest 2011;139:101-108.

-9 Ernst A, Feller-Kopman D, Becker HD, et al: Central airway obstruction. Am J Respir Crit Care Med 2004;169:1278-1297. 
10 Lin YC, Tsai YH, Huang CC, et al: Outcome of lung cancer patients with acute respiratory failure requiring mechanical ventilation. Respir Med 2004;98:43-51.

$\checkmark 11$ Chhajed PN, Baty F, Pless M, et al: Outcome of treated advanced non-small cell lung cancer with and without central airway obstruction. Chest 2006;130:1803-1807.

12 Colt HG, Harrell JH: Therapeutic rigid bronchoscopy allows level of care changes in patients with acute respiratory failure from central airways obstruction. Chest 1997;112: 202-206.

13 Shaffer JP, Allen JN: The use of expandable metal stents to facilitate extubation in patients with large airway obstruction. Chest 1998;114:1378-1382.

14 Jeon K, Kim H, Yu CM, et al: Rigid bronchoscopic intervention in patients with respiratory failure caused by malignant central airway obstruction. J Thorac Oncol 2006; 1: 319-323.

15 Stephens KE Jr, Wood DE: Bronchoscopic management of central airway obstruction. J Thorac Cardiovasc Surg 2000;119:289-296.
16 Schuster DP: Everything that should be done - not everything that can be done. Am Rev Respir Dis 1992;145:508-509.

17 Schapira DV, Studnicki J, Bradham DD, et al: Intensive care, survival, and expense of treating critically ill cancer patients. JAMA 1993;269:783-786.

18 Groeger JS, Lemeshow S, Price K et al: Multicenter outcome study of cancer patients admitted to the intensive care unit: a probability of mortality model. J Clin Oncol 1998;16: 761-770.

19 Soares M, Darmon M, Salluh JI, et al: Prognosis of lung cancer patients with life-threatening complications. Chest 2007;131:840846.

20 Roques S, Parrot A, Lavole A, et al: Sixmonth prognosis of patients with lung cancer admitted to the intensive care unit. Intensive Care Med 2009;35:2044-2050.
21 Stanopoulos IT, Beamis JF Jr, Martinez FJ, et al: Laser bronchoscopy in respiratory failure from malignant airway obstruction. Crit Care Med 1993;21:386-391.

22 Lo CP, Hsu AA, Eng P: Endobronchial stenting in patients requiring mechanical ventilation for major airway obstruction. Ann Acad Med Singapore 2000;29:66-70.

23 Vonk-Noordegraaf A, Postmus PE, Sutedja TG: Tracheobronchial stenting in the terminal care of cancer patients with central airways obstruction. Chest 2001;120:1811-1814.

24 Huskamp HA, Keating NL, Malin JL, et al: Discussions with physicians about hospice among patients with metastatic lung cancer. Arch Intern Med 2009;169:954-962.

25 Temel JS, Greer JA, Muzikansky A, et al: Early palliative care for patients with metastatic non-small-cell lung cancer. N Engl J Med 2010;363:733-742.

-26 Shelton BK: Admission criteria and prognostication in patients with cancer admitted to the intensive care unit. Crit Care Clin 2010; 26:1-20. 